\title{
Resistencia en el aula: entre el fracaso y la indignación
}

Resistance in the classroom: between failure and indignation

Elsie Rockwell ${ }^{1}$

\section{Resumen}

En este artículo abordo una disyuntiva conceptual en la explicación de la resistencia estudiantil en clase. Resumo algunas de las posturas clásicas acerca de la resistencia (WILLIS, GIROUX), retomando la distinción entre fenómenos que contribuyen al fracaso escolar de los estudiantes, y aquellos que muestran indignación. Discuto resultados de estudios que intentan explicar los momentos de discontinuidad en el flujo de la interacción en el aula como evidencia de resistencia de parte de los alumnos. Luego examino las implicaciones de las teorías de Basil Bernstein y de Jürgen Habermas para explicar estos procesos. Sugiero que no toda expresión de resistencia en el aula debe ser vista como señal de incompetencia, diferencia cultural o respuesta autocondenadora. Con base en la teoría de comunicación de Habermas, es posible comprender muchas respuestas de los estudiantes como legítima (si bien indirecta) invocación de las pretensiones de verdad, adecuación y veracidad.

Palabras-clave: Resistencia; Interacción en el Aula; Bernstein; Habermas; Teoría Crítica; Sociolingüística

\section{Abstract}

In this paper, I approach a conceptual dilemma involved in understanding student resistance in classrooms. I summarize some of the classic positions on resistance (WILLIS, GIROUX), and recall the distinction between behaviors that contribute to "school failure" and those that express "moral indignation". I discuss classroom studies that attempt to explain moments of discontinuity in the flow of verbal interaction as evidence of student resistance. I then turn to the implications of the theories of Basil Bernstein and Jürgen Habermas to explain these processes. I suggest that not all expressions of resistance in the classroom should be seen as signs of incompetence, cultural difference, or selfcondemning behavior. Drawing on Habermas' theory of communication, it is possible to understand many student's responses as a legitimate, albeit indirect, invocation of the validity claims of truth, correctness and sincerity.

Keywords: Resistance; Classroom Interaction; Bernstein; Habermas; Critical Theory; Sociolinguistics

\footnotetext{
1 Doctora en Ciencias en la especialidad de investigaciones educativas. Centro de Investigación y Estudios Avanzados, México. erockrich@yahoo.com.
} 
La resistencia ha sido un tema central de los estudios de culturas estudiantiles y procesos de interacción escolar durante varias décadas. Algunos investigadores han fundamentado sus argumentos en torno a la resistencia estudiantil en descripciones de culturas estudiantiles, particularmente aquellas que entran en conflicto con las reglas escolares. En este ensayo, examino investigaciones hechas desde la sociolingüística y el interaccionismo simbólico que han ofrecido herramientas más finas para comprender los mecanismos cotidianos de ese proceso. El análisis detallado de la interacción permite destacar los márgenes reales que tienen los estudiantes frente a los maestros y los límites que los maestros enfrentan al trabajar con grupos. Muchos de esos estudios suelen atribuir la resistencia estudiantil a la incompatibilidad entre la cultura escolar y ciertas disposiciones culturales expresadas, producidas o utilizadas por los estudiantes en situaciones de interacción con los maestros. Otras perspectivas, en particular la pragmática, sugieren que las diferencias culturales de los estudiantes se entretejen con principios universales de la comunicación, para producir acciones de resistencia ante el discurso escolar.

\section{El reto de la resistencia}

Durante los decenios de 1970 y 1980, describieron procesos de resistencia que permitieron cuestionar la eficacia que algunos teóricos le otorgaban al aparato escolar. Un hito en esa discusión fue el estudio realizado por Paul Willis (1977), quien introdujo el concepto de resistencia cultural y elaboró las distinciones y las vinculaciones entre ese proceso y los de producción cultural, reproducción cultural y reproducción social (1981). La teoría de la resistencia cultural permitió contrarrestar el peso de la teoría de la reproducción, particularmente en la versión del libro clásico de Pierre Bourdieu y Jean Claude Passeron (1981). A diferencia del postulado de estos autores, quienes sostuvieron que los estudiantes de clases bajas eran excluidos de la cultura escolar por no contar previamente con el capital cultural necesario para aprovechar la enseñanza escolar, Willis argumentó que los jóvenes estudiantes de clases trabajadoras en una ciudad inglesa producían una "contracultura escolar" que los llevaba a "optar" por ser obreros. Esta disposición cultural generaba una resistencia ante las reglas y las formas culturales de la escuela y permitía desenmascarar 
la ilusión de ascenso social que formaba parte del discurso ideológico de la escuela. Sin embargo, también contribuía al 'fracaso' escolar de esos estudiantes y, finalmente, a la reproducción de la estructura social.

A raíz del estudio de Willis, se dio una nutrida discusión en torno a la resistencia, dentro del campo denominado "la nueva sociología de la educación". Algunos textos matizaron y elaboraron los planteamientos iniciales. ${ }^{2}$ Varios estudios etnográficos retomaron y polemizaron con las conclusiones de Willis, y ofrecieron puntos de comparación con lo que pasaba en las escuelas en diferentes contextos sociales y culturales. ${ }^{3}$ Tomaban en cuenta los efectos de género y adscripción étnica o racial, así como las diferencias atribuibles a las condiciones desiguales de las escuelas. Algunos investigadores relacionados con el interaccionismo simbólico criticaban esos estudios argumentando que carecían de un análisis más cercano a los sujetos involucrados, tanto maestros como estudiantes, cuya capacidad de crear respuestas y estrategias inéditas en las escuelas era cada vez más evidente. ${ }^{4}$ Esto obligó a reformular los conceptos y a enfrentar los problemas metodológicos involucrados en tratar de observar el proceso de resistencia en estudios empíricos.

En esa línea, Frederick Erickson, antropólogo dedicado al estudio de las interacciones en el aula, intentó crear una explicación que vinculara las posturas de la nueva sociología con los estudios sociolingüísticos en el aula. Para ello consideró la cuestión del poder.

El poder, como capacidad de coacción sobre otros, pertenece potencialmente tanto a los maestros como a los alumnos en el aula. La autoridad, como uso legítimo del poder y foco del conocimiento y del juicio socialmente sancionado, reside oficialmente con el maestro. La influencia, como capacidad no sancionada de ejercer el poder, reside con los alumnos. [...] El ejercicio de la autoridad (por el maestro) en ausencia del consentimiento de los alumnos puede cuando mucho conducir a un conformismo superficial con la

2 APPLE (1981), BOURDIEU (1988), WILLIS (1981), GIROUX (1985), McCARTY y APPLE (1988), WEIS (1988).

3 SHARP y GREEN (1975), ANYON (1981), FOLEY (1990), CONNELL et al. (1982), EVERHART (1983), TADEU DA SILVA (1988). Para una discusión más reciente, ver LEVINSON y HOLLAND (1996).

4 HARGREAVES (1978), HAMMERSLEY (1977), WOODS (1980). 
voluntad del maestro; es decir, en la situación de enseñanza los alumnos siempre poseen la capacidad de resistir negándose a aprender lo que el maestro intenta que aprendan... ${ }^{5}$

Erickson mostró que el fracaso escolar puede ser consecuencia de esa resistencia micropolítica, que si bien resulta ser autocondenadora, no corresponde a una falta de capacidad o de motivación por parte de los alumnos. Erickson también relacionó las diferencias culturales a la micropolítica del aula, sugiriendo que:

Como fuente de dificultades interaccionales relativamente pequeñas, las diferencias culturales pueden convertirse no obstante en recursos para la construcción de conflictos a mayor escala y difusión, entre maestros y alumnos. En este sentido las diferencias culturales correspondientes a clase social, etnicidad, raza, género, o condiciones de discapacidad (bandicapping), pueden intervenir en la creación de aquellas situaciones en las que algunos alumnos se niegan a aprender, como forma de resistencia frente a los maestros. ${ }^{6}$

Esta interpretación de la dinámica en el aula es cercana a la perspectiva planteada por Willis (1977), ya que se trata de un tipo de resistencia que conduce al fracaso escolar. Sin embargo, a diferencia de aquel estudio, Erickson enfatizó el papel activo de la escuela en la producción de barreras comunicativas y situaciones de discriminación.

En un artículo clave, Henry Giroux señaló que no toda manifestación de oposición puede ser considerada evidencia de la resistencia. Propuso crear una noción más rigurosa de resistencia que permitiera encontrar, entre todos los fenómenos de oposición, "aquéllos que realmente estén ligados a la indignación política o moral, y no sólo a la desviación o la impotencia aprendida". Agregó: "En la medida en que las conductas de oposición suprimen las contradicciones y simultáneamente se funden con, en lugar de retar, la lógica de la dominación ideológica, no corresponden a la categoría de la resistencia, sino a su contrario, la acomodación y el conformismo". ${ }^{8}$ Plantea así el reto de distinguir entre actos que se deben a la indignación y los que abonan la lógica de la reproducción social. En

5 ERICKSON (1986, p. 137, traducción de la autora).

Ibidem, p. 138 (traducción de la autora).

GIROUX (1985, p. 289).

Ibidem, p. 290. 
este ensayo, intento mostrar cómo se puede expresar la resistencia en la escala cotidiana, particularmente cuando el flujo de comunicación entre maestro y alumnos se dificulta o se interrumpe. Para considerar esos momentos, presento diversos aportes teóricos que muestran que los sujetos recurren a elementos culturales, de diversa constitución histórica, que corresponden ya sea a tradiciones propias o bien a estructuras culturales genéricas que subyacen a la lengua y a la cognición de todo ser humano. Sugiero que algunas perspectivas poco exploradas, particularmente la pragmática de Habermas, permiten apreciar actos de resistencia que se deben a la indignación.

\section{ANÁlisis SOCIOLINGÜÍSTICOS DE LA INTERACcIón ESCOLAR}

No es tarea fácil distinguir los procesos de resistencia implicados en la interacción verbal. Siempre existe el riesgo de tomar el contenido del discurso oral como evidente en sí mismo, sin considerar los múltiples niveles de inferencia en juego en la comunicación. ${ }^{9}$ No es posible confiar en un análisis del contenido explícito de los enunciados, sin tomar en cuenta la complejidad de la interacción comunicativa. Cada perspectiva teórica utilizada lleva a una interpretación distinta de la lógica social o cultural subyacente a la interacción.

La interacción entre maestro y alumnos ha sido estudiada por investigadores desde varias corrientes de la sociolingüística y de la etnometodología. ${ }^{10}$ Los estudios iniciales sostenían que las estructuras de participación y las reglas de uso correspondientes a la interacción en el aula eran diferentes de las que predominaban en otros espacios sociales, argumentando que en el aula generalmente es el maestro quien retoma la iniciativa en el turno siguiente al de cualquier alumno. ${ }^{11}$ Se consideraba que la asimetría del poder característica de la escuela tenía consecuencias en las pautas del intercambio verbal en el aula.

La naturaleza de las preguntas en el aula ofrecía evidencia de estas consecuencias. Bajo la forma gramatical de la pregunta se encuentran

\footnotetext{
9 STUBBS (1983), DURANTI (2000).

10 CAZDEN, JOHN y HYMES (1972), PHILIPS (1972), CICOUREL et al. (1974), HAMMERSLEY (1977), McDERMOTT (1977), MEHAN (1979), HYMES (1980), ERICKSON (1982), HEATH (1983), STUBBS (1983), EDWARDS y MERCER (1987), CAZDEN (1988), HICKS (1996), entre otros.

11 ATKINSON (1981, p. 107), STUBBS (1983, p. 29, 63), ERICKSON (1982).
} 
órdenes indirectas, que se responden con la ejecución de una acción; también aparecen preguntas como elementos retóricos en el monólogo del maestro, en cuyo caso no se espera una respuesta verbal. Cuando los maestros esperan una respuesta verbal por parte de los alumnos, a menudo hacen una especie de "pseudopregunta"; 12 no se trata de una pregunta para averiguar algo nuevo, sino de una pregunta de "información conocida", cuya respuesta se evalúa según algún criterio de lo correcto. En esas situaciones no siempre es evidente para los alumnos cómo deben contestar, de tal manera que ellos hacen varios intentos de interpretar lo que espera el maestro para poder producir una respuesta aceptable. ${ }^{13}$

En un estudio temprano, Mehan (1979) adoptó un enfoque que llamó "etnografía constitutiva", para examinar clases videograbadas en busca de la estructura de las secuencias de comunicación. Utilizó una serie de categorías de tipo generativo, que descomponían la clase, como unidad mayor, en una serie de "fases" constituidas a su vez por "conjuntos tópicos". Dentro de éstos, aisló "secuencias" de interacción verbal, en las cuales encontraba que la unidad básica tenía una estructura de "iniciaciónrespuesta-evaluación" (IRE). Mehan observó que el intercambio mínimo solía consistir en una "interrogación" del maestro, seguida por una respuesta del alumno y luego un comentario evaluativo del maestro. Propuso que en esas estructuras operaban restricciones a las reglas que normalmente distribuyen los turnos entre los participantes en una conversación. ${ }^{14}$

Mehan se proponía comprender las reglas que deben adquirir los estudiantes para poder participar con éxito en las interacciones estructuradas de esa manera. El modelo suponía que la escuela socializa a los alumnos en el uso de estas reglas, y cualquier respuesta errónea se consideraba consecuencia de una "falla" en el proceso de socialización. $\mathrm{La}$ "competencia comunicativa" requerida para participar en clase presuponía aprender no solamente el conocimiento que transmite el maestro, sino también las maneras de formular (cast in a form) el conocimiento adecuadamente dentro de las estructuras de interacción. ${ }^{15}$ 
La desvinculación entre el contenido y la forma de enunciación explicaba, según Mehan, ${ }^{16}$ las intervenciones inapropiadas de los alumnos. Sin embargo, el modelo no consideraba que ciertas respuestas consideradas inadecuadas, en lugar de indicar una falta de competencia, puedan significar una expresión de resistencia.

El desarrollo de perspectivas más dinámicas sobre lo que ocurre en la comunicación llevó a una descripción distinta de la interacción en el aula. Una primera aproximación enfatizó las diferencias culturales en las formas de comunicación, que incluían no solamente estructuras lingüísticas sino también rasgos paralingüísticos, como el hecho de establecer o no contacto visual con un interlocutor. También se estudiaron las normas que rigen la comunicación oral en diferentes culturas, y que explican, por ejemplo, la reticencia de alumnos indígenas a hablar frente a la clase, a pesar de que en pequeños grupos solían expresarse da manera fluida. ${ }^{17}$ Por otra parte, el análisis conversacional de orientación etnometodológica centraba la atención no en los antecedentes de los hablantes sino en lo que éstos construían en la situación. Se estudiaban las formas en que se logra (achieve) una conversación, ${ }^{18}$ considerada como un sistema manejado por los participantes, es decir una forma comunicativa "trabajada" sobre la marcha y generada gracias a la colaboración entre interlocutores. ${ }^{19}$ Ese enfoque permitió ver cómo se "lograban", o no, los diversos intentos de comunicación en el aula. ${ }^{20}$ Finalmente, la integración de varias corrientes lograda por John Gumperz (1982) permitió observar que maestros y estudiantes continuamente están utilizando los recursos discursivos que poseen para renegociar la definición misma de la situación y de las reglas que la rigen.

Si se acepta, como lo planteó Erickson, que toda interacción está inserta en relaciones de poder, la interacción en el aula cobra otras dimensiones. La cuota variable de poder que tienen los maestros les permite imponer, legítimamente, tanto pautas de interacción como interpretaciones y delimitaciones del contenido en el aula. Aunque, en los estudios iniciales, 
rara vez se constataba la iniciativa que muchos alumnos toman en la interacción, sí se notó que ejercen cierto poder. ${ }^{21}$ Actualmente, muchos estudios han mostrado que durante las clases, tanto maestros como alumnos utilizan estrategias que conducen a la negociación de lo que realmente se hace y se dice. La naturaleza estratégica de la interacción significa que ni unos ni otros siguen reglas ni rutinas fijas, establecidas de antemano. Los maestros cuentan con recursos discursivos que les permiten "guiar" a los alumnos hacia la respuesta correcta, retractarse de una afirmación o de una pregunta que los alumnos mismos detectan como incongruente o errónea, abrir o cerrar temas legítimos y comunicar a los alumnos los límites de su autonomía. Por otra parte, los alumnos cuentan con poderosas armas contra las intenciones del maestro, como el silencio y el ruido, cuyo efecto en lograr la suspensión o el cambio de rumbo de una actividad o secuencia en la clase no es despreciable. ${ }^{22}$

Las estrategias desplegadas en la interacción en el aula producen efectos significativos. Los docentes intentan asegurar la continuidad de la interacción con los alumnos, ya que deben contar con su participación para la realización misma de su trabajo. No obstante, las intervenciones de los maestros tienen un alto grado de "indexicalidad", en términos etnometodológicos, ${ }^{23}$ es decir tienen palabras o frases cuyo significado es necesario inferir de algún elemento del contexto social o discursivo. Las estrategias de los alumnos se orientan hacia la interpretación de las expresiones explícitas o implícitas del maestro, en busca de la respuesta correcta a sus solicitudes. Los maestros por su parte procuran encauzar los procesos de inferencia mediante el uso de claves o señales de contextualización (contextualization cues), como mostraba Gumperz (1982).

Esas estrategias pueden llevar al consabido juego de "seguir las pistas que da el maestro", o a lo que Jackson (1968) identificó como una "simulación del aprendizaje". En ciertos momentos, se producen con relativa facilidad las respuestas esperadas y se continúa con la clase, independientemente de la comprensión de los temas. De hecho, es bastante frecuente encontrar que el contenido puede ser irrelevante en algunas prácticas recurrentes en la escuela, como los cuestionarios, el dictado, la

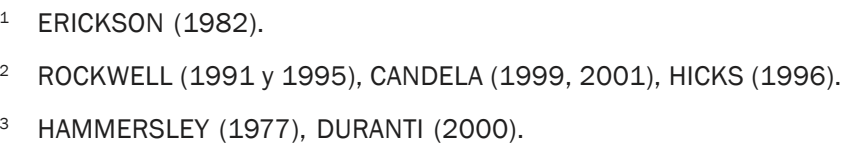


recitación y el análisis gramatical. ${ }^{24}$ En esos casos, se da una disociación entre lo que hemos llamado ${ }^{25}$ la "lógica de la interacción", a la cual deben atender los alumnos para poder responder de la manera esperada, y la "lógica del contenido" del texto o del discurso docente. Una disociación similar fue notada por Erickson, bajo los conceptos complementarios de la "estructura de participación social" y la "estructura de la tarea académica"26, distinción que fue retomada por Cazden (1988).

No obstante, los estudiantes también tienen la posibilidad de evitar ese proceso de simulación. Al atender simultáneamente a la lógica de la interacción y a la lógica del contenido, los alumnos a veces producen intervenciones o respuestas que pueden ser vistas como "errores" por los maestros, sobre todo cuando las dos lógicas no coinciden. En ocasiones los alumnos siguen la "lógica del contenido", por ejemplo al leer el libro, para llegar a sus propias conclusiones, que no siempre son las previstas por el docente. En ese momento, los maestros reaccionan de diferentes maneras, aceptando, reformulando o rechazando las respuestas de los alumnos. $^{27}$

Los análisis anteriores reconstruyen en gran medida sólo aquella parte de la interacción que corresponde a las clases formales, en las que se pueden identificar pautas escolares recurrentes sin considerar el "ruido". Es posible pensar en esta parte de la interacción como el dominio público del aula. Sin embargo, a partir de otros estudios etnográficos se observa que en toda aula también hay un dominio privado, en el que se dan múltiples interacciones, así como intervenciones simultáneas de varios hablantes. Esas situaciones ocurren sobre todo entre los alumnos, pero a veces también entre el maestro y los alumnos. ${ }^{28}$

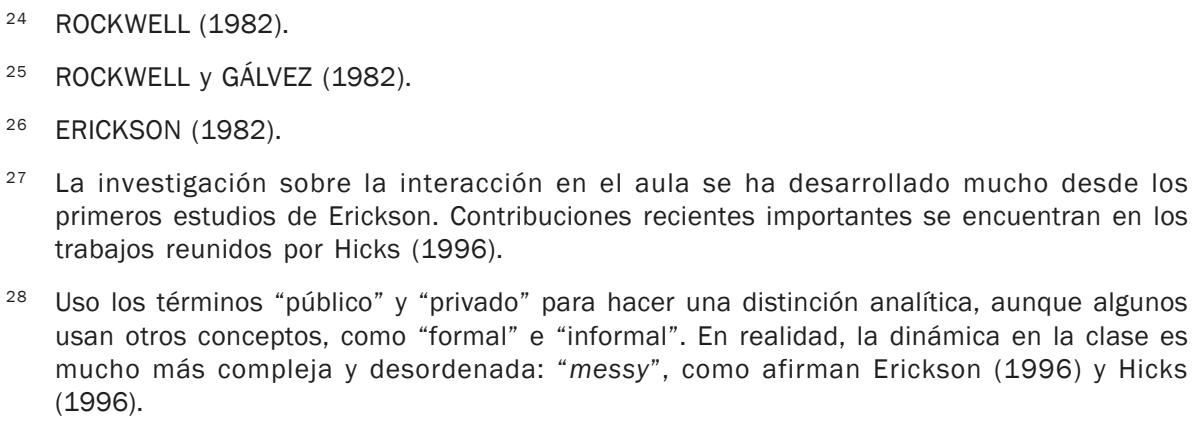

28 Uso los términos "público" y "privado" para hacer una distinción analítica, aunque algunos usan otros conceptos, como "formal" e "informal". En realidad, la dinámica en la clase es mucho más compleja y desordenada: “messy”, como afirman Erickson (1996) y Hicks (1996). 
Esos dos dominios no son exclusivos el uno del otro. ${ }^{29}$ Continuamente aparecen en el dominio privado contenidos presentados en el dominio público que los alumnos se apropian y reelaboran. Por otra parte, hay contenidos producidos en el dominio privado que irrumpen en el dominio público delimitado por el maestro, aun dentro de las estructuras de participación que enmarcan esa interacción. En el ámbito privado los alumnos siempre expresan reacciones, comentarios o sugerencias que el maestro puede retomar o ignorar, si es que se percata de ellos. Ambos dominios de hecho son constitutivos de una misma realidad cotidiana en el aula. En los procesos de apropiación del conocimiento en el aula cuentan tanto las interacciones formales o públicas con el maestro, como las interacciones que ocurren entre alumnos en torno a las tareas escolares, muchas de las cuales son asignadas a la categoría escolar de "copiar". 30

Los contenidos de esas interacciones, que son de difícil acceso al investigador, no sólo por razones técnicas (de registro) sino también por razones conceptuales, sugieren que en el dominio "privado" se generan algunos de los procesos de resistencia más interesantes en el aula. ${ }^{31} \mathrm{Un}$ ejemplo se observó en una clase donde niños indígenas resolvían ejercicios matemáticos en su lengua materna, en "privado", y luego discutían entre ellos el problema de quién iba a exponer, en el dominio público y en castellano, los resultados de la tarea. ${ }^{32}$ En ese caso, la interacción entre los niños, si bien puede verse como "oposición” a las normas de la escuela que requieren el uso de español, se dio en función de la apropiación del conocimiento escolar, lo cual difiere de la interpretación usual de la resistencia como el hecho de "negarse a aprender". 33

La noción de resistencia toma otro sentido en el trabajo de Hervé Varenne y Ray McDermott. Al insistir en analizar cómo se constituyen las trayectorias hacia el "fracaso escolar", ellos proponen que la resistencia es un proceso constante, común tanto a maestros como a estudiantes de diversas clases sociales, frente a la "cultura" competitiva dominante de la

\footnotetext{
29 Kris Gutiérrez y colaboradores (1995) Ilamaron los dos espacios "guión” y "contra-guión" (script, counterscript) y propusieron un espacio intermedio o 'tercer espacio', en el cual se entrecruzan contenidos de un lado y de otro.

30

ROCKWELL (1982).

ROCKWELL (1991), CANDELA (1999).

VALENTÍNEZ (1982).

ERICKSON (1986).
} 
escuela estadounidense. Recuerdan que la organización escolar presupone nichos que se tienen que llenar con estudiantes "fracasados", y por lo tanto se ponen en marcha múltiples mecanismos cotidianos de identificación y trato diferencial. No obstante, al enfatizar el carácter activo de los sujetos involucrados en el proceso escolar, los autores subrayan su capacidad para "extender los límites de las condiciones dadas." ${ }^{34}$ En esta perspectiva, la resistencia no contribuye al fracaso escolar, más bien intenta contrarrestar los mecanismos escolares que lo producen.

Las aproximaciones a la interacción en el aula revisadas en esa sección muestran facetas diversas, pero complementarias, de situaciones de comunicación escolar que son muy complejas. Para el estudio de la resistencia, es particularmente significativa la interpretación de los momentos problemáticos donde se interrumpe el flujo de comunicación entre maestros y alumnos. Analizar esos momentos requiere ver más allá de las deficiencias o diferencias en las competencias comunicativas de los estudiantes, para intentar comprender acciones estratégicas y relaciones de poder que no son fáciles de descifrar.

\section{De la resistencia autocondenadora}

\section{A LA RESISTENCIA COMO INDIGNACIÓN}

Para profundizar en la comprensión de la resistencia en el aula, comparo las implicaciones de dos teorías, que si bien han sido referentes constantes en la discusión acerca de la educación en América Latina, no han tenido el mismo grado de integración a la investigación empírica en el aula que las perspectivas sociolingüísticas consideradas anteriormente. Argumento que a diferencia del modelo de competencias comunicativas de Basil Bernstein, que subraya las diferencias entre clases sociales, la pragmática de Jürgen Habermas permite identificar actos de resistencia basados en estructuras comunes a todo hablante, aun reconociendo las desigualdades estructurales de la sociedad.

Abordar la teoría de Bernstein (1971 y 1975) cobra sentido por la fuerte influencia que ha tenido sobre investigadores y educadores en América Latina. No obstante, es importante recordar que Bernstein es sociólogo de formación y orientación, y sus análisis de aspectos del uso

34 VARENNE y McDERMOTT (1999, p. 126 y cap. 6). 
del lenguaje carecían del rigor esperado por los lingüistas, que en general no avalaron del todo sus conclusiones. ${ }^{35}$

Bernstein, partiendo de una concepción durkheimiana de la socialización, formalizó un modelo que sitúa las dos orientaciones básicas postuladas en sus escritos iniciales - el código elaborado y el código restringido - en el campo de las relaciones de dominación y los dispositivos de control simbólico. Bernstein (1981) definió los códigos como mecanismos culturalmente determinados (culturally determined positioning devices) que colocan a los sujetos en cierta posición respecto a las formas dominantes y dominadas de comunicación. En sus términos, esos códigos son "principios regulativos, adquiridos tácitamente", que operan como estructuras semánticas y sintácticas subyacentes. Son constitutivas de la competencia comunicativa que se adquiere mediante las inferencias que el sujeto hace a partir de los rasgos de una interacción cotidiana continua, especialmente en la familia. Los dos códigos se caracterizan sobre todo por una oposición entre la "independencia" y la "dependencia" respecto a contextos particulares. ${ }^{36}$ Así, en la versión de Bernstein el 'código restringido' produce cierto tipo de enunciados cuya comprensión está estrechamente ligada al contexto de comunicación. En cambio el 'código elaborado' permite formular enunciados cuya interpretación no depende del contexto inmediato. ${ }^{37}$ Bernstein asoció el 'código restringido' con las clases trabajadoras, y consideró que sólo las clases 'altas' tenían acceso al código elaborado desde su infancia.

Bernstein usó los conceptos de clasificación y de enmarcación para relacionar la competencia comunicativa y la estructura social. En su teoría, la clasificación se mantiene y se legitima al reproducirse el aislamiento (insulation) entre las categorías o grupos sociales creados por la distribución del poder ${ }^{38}$ El aislamiento a su vez se mantiene por la "no comunicación", es decir por la discontinuidad entre las "voces" de diferentes categorías

35 HYMES (1972), STUBBS (1976).

36 BERNSTEIN (1981, p. 327-331). Ver también traducciones de algunos de sus artículos en la Revista Colombiana de Educación 15, 1985.

37 Los lingüistas han hecho notar que todo hablante usa un 'código restringido' en algunos contextos de comunicación oral, y que el discurso 'elaborado', tal como lo ejemplifica Bernstein, también depende de contextos específicos. Señalan que Bernstein no distinguió entre la lengua oral y la lengua escrita y que, en todo caso, es en lo escrito que se encuentran algunos de los rasgos que él asocia con el 'código elaborado'.

BERNSTEIN (1981, p. 333-335). 
sociales. ${ }^{39}$ Mediante la "enmarcación", por otra parte, se establecen los mecanismos de control que aseguran la distribución desigual de los recursos discursivos en las sociedades de clase y la reproducción de las orientaciones básicas hacia el dominio simbólico o ideológico.

Bernstein consideró que en el discurso escolar predomina el código elaborado, generalmente con una fuerte clasificación y enmarcación..$^{40}$ En su perspectiva, los alumnos provenientes de las clases trabajadoras se caracterizan como "discapacitados" por poseer sólo el código restringido. La reproducción familiar de ese código refuerza la reproducción de la estructura de clases, al no permitir que los alumnos se apropien del código elaborado y de los conocimientos correspondientes. La restricción del acceso al conocimiento expresado mediante el código elaborado en la escuela contribuye al aislamiento entre las categorías sociales en la estructura del poder. ${ }^{41}$

Bernstein previó como posibilidades del código restringido ciertos procesos de resistencia; afirmó: "la variación, la resistencia, el reto, la oposición o la lucha, surgen de la relación estructural producida por la clase misma". ${ }^{42}$ No obstante, en su opinión ésas son resistencias producidas dentro del dominio del código restringido y no logran modificar la clasificación y la enmarcación. ${ }^{43}$ Nuevamente, se trata de una resistencia que se revierte sobre los propios sujetos, sin afectar la distribución del poder. En este sentido, aunque Bernstein no lo afirme, esta noción de resistencia también se encuentra al servicio de una reproducción autocondenadora.

La perspectiva de Bernstein ayuda a evitar la tendencia de ver "resistencias" en todas las oposiciones cotidianas a la autoridad del maestro y de suponer a partir de ello cambios fundamentales en la distribución del poder en la sociedad. Sin embargo, no da la posibilidad de distinguir resistencias que se deban a la indignación, y no a la desventaja cultural o la incompetencia frente a las estructuras comunicativas y sociales dominantes en las escuelas.

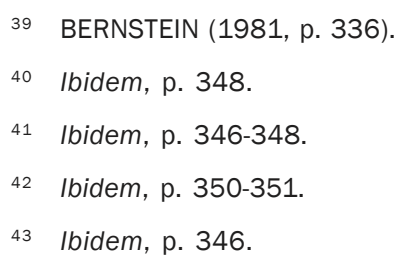


Algunos conceptos de la pragmática, disciplina que abordó la comunicación inicialmente desde preceptos filosóficos, permiten aventurar interpretaciones distintas de lo que ocurre cuando se dan discontinuidades en la interacción verbal entre maestro y alumnos. Es particularmente útil la formulación de las 'pretensiones de validez' que propuso Jürgen Habermas. Aunque no exista un cuerpo de investigación empírica desde esa perspectiva, la alternativa teórica abre la posibilidad de comprender ciertos incidentes como evidencia de procesos de resistencia, en función de principios comunes a la competencia comunicativa de todo hablante y no de diferencias radicales entre orientaciones, códigos o competencias de los estudiantes y de los maestros.

Uno de los avances importantes de la pragmática fue el concepto de "acto de habla", unidad mínima de análisis de uso del lenguaje, cuyas propiedades no se pueden derivar en su totalidad de la estructura gramatical de las expresiones lingüísticas utilizadas. A partir de la constatación de que en los actos de habla "gramaticalmente se dice menos de lo que se significa", se buscaron elementos contextuales y conversacionales que explicaran el significado de cada acto. Esos análisis formales contribuyeron a la concepción del uso del lenguaje en términos de acciones, de lo que "hacemos" al hablar de determinada manera (ordenar, prometer, amenazar, bautizar, afirmar, negar, constatar, entre otros). ${ }^{44}$

Desde esa perspectiva, un planteamiento importante fue que todo enunciado tiene una doble estructura, ${ }^{45}$ consistente en el "contenido proposicional" por una parte, y la "fuerza ilocucionaria" por otra. La fuerza ilocucionaria generalmente compromete al hablante o al oyente a ser consecuentes con determinada acción. Eso es evidente en el caso de las promesas o las órdenes, pero también por ejemplo en cualquier expresión lingüística cuya función es interrogativa (aunque no tenga la forma gramatical de una pregunta), en la medida que compromete al oyente a responder. En cualquier acto de habla el oyente no solamente debe comprender el contenido proposicional, sino que también debe interpretar lo que el hablante está "haciendo" al decir determinada cosa. La reflexividad inherente a todo enunciado, dada esa doble estructura, obliga a considerar la inferencia que el oyente debe hacer acerca del sentido de

44 AUSTIN (1962), SEARLE (1969), LEVINSON (1983), DURANTI (2000).

45 RECANATI (1979). 
la fuerza ilocucionaria, ${ }^{46}$ aun en ausencia de marcas gramaticales (como "te prometo" o "te ordeno"). La interpretación de ese sentido es necesaria para asegurar la aceptación del acto por el oyente, de tal manera que la acción comunicativa llegue a "feliz término", y no sea interpretada erróneamente.

En principio, la insistencia de la pragmática en considerar que una parte de la significación se produce en los actos de habla, y no es inherente a las estructuras gramaticales y semánticas en sí, permite cuestionar concepciones que presuponen algún manejo en abstracto de códigos sintácticos o semánticos como condición para la apropiación de significados o conocimientos escolares. Incluso en aquellas situaciones en que no son compartidos los referentes culturales y convencionales, a menudo se tiene alguna interpretación acerca del sentido ilocucionario de lo dicho. Por ejemplo, a veces es posible inferir que lo que alguien dice es una orden o un regaño aun sin entender la lengua en que se expresan. De hecho, Gumperz (1982, p. 6-8) ha argumentado que a menudo las diferencias culturales y lingüísticas facilitan la comunicación; no siempre constituyen obstáculos al entendimiento.

Desde esa perspectiva, sería importante explorar significados generados en la interacción cotidiana en el aula, independientemente de las diferencias culturales entre los hablantes. En principio los estudiantes, incluso aquellos de las clases trabajadoras o con antecedentes culturales distintas a las pautas escolares, pueden y suelen interpretar el discurso en el aula de alguna manera. Sería posible retomar y comprender el sentido de esas apropiaciones por parte de los alumnos, sin soslayar su importancia con argumentos derivados de la falta de competencia o de la incompatibilidad de pautas culturales.

Siguiendo otra lógica, y como parte de su amplia propuesta filosófica y sociológica, Habermas (2000, p. 351-432) exploró formas de desligar el análisis de la fuerza ilocucionaria de los procesos de inferencia conversacional relacionados con elementos contextuales. Propuso desarrollar una teoría estructural de los actos de habla que no dependiera

46 La pragmática reconstruye procesos de inferencia propios de todo oyente en cualquier situación de interacción verbal. En este caso, las infracciones contra el proceso normal de inferencia, así como la ironía, los chistes, entre otros, proporcionan el material para explorar esos procesos, que se han planteado desde diferentes perspectivas (Austin, Grice, Searle, Recanati, entre otros). 
de las intenciones o los conocimientos antecedentes de los sujetos ni de las normas de instituciones o culturas particulares, aunque reconocía que esas condiciones intervienen en situaciones concretas de comunicación. Su intención era encontrar propiedades formales universales de los actos de habla. ${ }^{47}$

La teoría de Habermas define como acción comunicativa sólo aquella orientada a lograr el entendimiento o acuerdo entre los interlocutores. La distingue de la acción estratégica, expresada en actos de habla orientados a lograr cierto efecto en el oyente, como lograr que éste diga o haga lo que el hablante quiere. La aceptación de cualquier acto de comunicación (no estratégico) depende del reconocimiento implícito de las 'pretensiones de validez', que de hecho se expresan mediante la fuerza ilocucionaria de cada acto de habla. ${ }^{48}$ Habermas retomó de la pragmática tres pretensiones de validez: "la verdad", "la rectitud" y "la veracidad". La "verdad" implica que el hablante dice lo que cree estar ajustado a la realidad y que puede argumentarlo con hechos. La "rectitud" supone que es correcto que el hablante emita cierto tipo de enunciado adecuado al contexto normativo, es decir, que no está "fuera de lugar". La "veracidad" lo compromete a ser consecuente con lo que dice, a ser sincero. En toda acción comunicativa, los hablantes tienen una especie de obligación de poder proporcionar las pruebas de la verdad, así como de justificar la rectitud del acto de habla, en caso necesario. Además deben mostrar la veracidad de sus palabras en las acciones subsecuentes a las que les compromete el acto de habla. ${ }^{49}$

En la acción comunicativa, el hecho de que el oyente no acepte lo dicho es una especie de apelación a su derecho de exigir que el hablante haga explicitas las bases en que se apoya el hablante para proponer implícitamente la verdad y la rectitud de lo que dice. En esos momentos, quien escucha puede mostrar desconfianza de la certeza de lo que ha dicho el hablante o de su derecho a decirlo, o bien dudar de su sinceridad.

47 A pesar de ese énfasis en lo universal, Habermas no pudo evitar cierto eurocentrismo al identificar la comunicación sincera con las formas históricas privilegiadas por el desarrollo de la "esfera pública" durante la época moderna en Europa. Es necesario romper con ese supuesto de su teoría, tomando en cuenta nuevamente la variación cultural e histórica de las formas de habla.

48 HABERMAS (2000, p. 394-400).

49 En la acción estratégica, en cambio se suspenden las pretensiones de validez, especialmente la "veracidad". 
Entonces, se desvía o interrumpe la comunicación y el hablante debe justificar lo que ha dicho, aunque sea indirectamente, para poder continuar.

Para construir su argumentación, Habermas eliminó todos aquellos elementos que podrían complicar el análisis formal, incluyendo las determinaciones institucionales y las acciones estratégicas. El esquema como tal es un modelo que permite aislar ciertas relaciones constantes, pero no pretende apresar en toda su complejidad las situaciones reales de interacción comunicativa. Eso hace particularmente difícil la utilización de esa perspectiva en la investigación empírica, necesaria para poder aclarar su pertinencia a la situación de interacción escolar. No obstante, Habermas recomendó utilizar su esquema en la investigación, recuperando necesariamente una serie de elementos, que incluyen relaciones interpersonales, convencionales, concretamente formadas en cada cultura, realizaciones lingüísticas implícitas, contingentes e indirectas (que deben inferirse del contexto), simultaneidad de orientaciones cognitivas y expresivas, así como la aparición de elementos estratégicos dentro de la acción comunicativa. La restricción de no considerar los actos determinados institucionalmente también tendría que modificarse al analizar la interacción escolar. ${ }^{50}$

Lo importante de ese modelo ideal es que la posibilidad de invocar las pretensiones de validez se considera un principio inherente a la competencia comunicativa de todo hablante, independientemente de la variante lingüística o los géneros discursivos que maneja. La estructura formal de cualquier acción comunicativa, considerada por Habermas como componente universal de la competencia comunicativa, se expresa de diferente manera en cada situación comunicativa y en cada lengua. Lo que interesa aquí es retomar la posibilidad de fundamentar una interpretación de las discontinuidades - incomprensiones, interrupciones, suspensiones - discursivas observadas en el aula en estructuras comunicativas comunes a los interlocutores. Es decir, es posible que ciertos momentos en el diálogo entre maestro y alumnos, en lugar de reflejar la "falta de comunicación" debida a códigos, competencias u orientaciones comunicativas diferentes, expresan más bien una apelación por parte de los alumnos frente a las pretensiones de validez, sobre todo de verdad y de rectitud de lo enunciado por el maestro. La no aceptación de lo enunciado en el aula, que los alumnos manifiestan más por vías indirectas

50 HABERMAS (2000, p. 419-432). 
que por oposición directa, puede indicar un cuestionamiento hacia la verdad del contenido proposicional, en lugar de una incomprensión de lo dicho o lo solicitado por el maestro. También puede significar que los alumnos no le reconocen al maestro el derecho a decir determinadas cosas ${ }^{51}$ o bien que dudan de su veracidad de lo que dice, de su sinceridad.

Esta perspectiva modifica la interpretación de aquellas discontinuidades que ocurren en la interacción verbal que se intentan usar como evidencia de resistencia por parte de los alumnos. De hecho, hay momentos en que la oposición sutil a lo dicho por el maestro, o la negativa a participar en la ritualizada y previsible interacción en clase, parece basarse en ese tipo de cuestionamiento, más que en la incompatibilidad cultural o la rebeldía autocondenadora. La apelación implícita a una pretensión de validez podría constituir un criterio para identificar momentos de resistencia que se basaran en la indignación. Para poder estudiar estas situaciones, se requieren nuevas herramientas, en particular las que ofrece el análisis conversacional para identificar turnos en que los hablantes aceptan o rechazan implícitamente lo dicho por otro, o bien reformulan o se retractan de lo que ellos mismos han dicho. ${ }^{22} \mathrm{Sin}$ pretender realizar este tipo de análisis, por el momento sólo ofrezco dos ejemplos sugerentes para ilustrar el argumento teórico.

En una clase registrada por Valentínez (1982), se encuentra un ejemplo claro. Los niños cuestionan la orden que dio el maestro de recolectar ciertas plantas, lo cual es trabajo de niñas; las niñas cuestionan lo correcto de que las mujeres vayan al monte, pues lo consideran una actividad que corresponde a los niños. Además, todos rechazaban la actividad propuesta por el maestro de plantar ciertos coditos o ramitas en el jardín escolar, diciendo que en ese tiempo no prenderían, por no ser "emenda" (tiempo de lluvias) y por haber heladas.

Este intercambio se puede interpretar como evidencia de una resistencia cultural, ya que se basa en elementos de una tradición cultural alternativa a la escolar (los niños son purépechas y recurren a sus conocimientos locales). Lo importante es que no se trata de un caso en que se suspende la comunicación porque los niños no comprenden lo que dice el maestro. Más bien, es una situación en que las diferencias culturales

51 Por ejemplo, en ciertas comunidades indígenas, sólo los parientes tienen derecho a regañar a un menor, por lo cual hay oposición a los maestros "regañones".

52 DURANTI (2000, cap. 8); CANDELA (2001). 
son puestas en juego sobre la base de supuestos compartidos acerca de lo que constituye un acto de habla aceptable. Los niños y las niñas cuestionan la "rectitud" de lo que solicita el maestro (exigen implícitamente que justifique su derecho a pedirles que recolecten ciertas plantas o que vayan al monte) y la verdad del contenido proposicional (que demuestre, frente a la certeza que tienen acerca de la imposibilidad de plantar en ciertas épocas, la verdad de lo que les enseña).

Aunque las diferencias culturales intervienen en esa secuencia, no impiden que los alumnos comprendan el contenido o infieran el sentido de lo que les pide el maestro. No se trata de desconocer las reglas del juego o las estructuras semánticas, ni de estar limitados por contar sólo con un 'código restringido'. La diferencia no es obstáculo a la comunicación, más bien proporciona las bases para dudar de lo dicho. La oposición en ese caso se fundamenta en estructuras comunicativas que los niños comparten con el docente.

Por otra parte, la reproducción del conocimiento cultural por parte de los alumnos en este caso, a diferencia de otros, no los lleva a una complicidad con los procesos de reproducción dominantes (por ejemplo, la discriminación o el racismo) ni a la autocondenación al fracaso (salvo que el docente les baje la calificación por no obedecer). En lugar de simplemente negarse a aprender lo que el maestro enseña, los alumnos se oponen a su intervención argumentando una mayor comprensión del contenido "verdadero" que el maestro pretende enseñar.

El mismo proceso parece ocurrir en otra clase, en la que no intervienen las diferencias culturales entre maestro y alumnos, pues ambos son de la localidad. El grupo de niñas de quinto grado presente en la clase (los niños están en deportes) se niega en ciertos momentos a continuar la interacción con el maestro, que consiste en responder a una serie de preguntas muy ritualizadas y llenas de pistas, acerca de un texto que han leído. La negativa a participar, aun siendo fácil inferir la respuesta deseada, se da en momentos en que el contenido solicitado contradice su experiencia cotidiana. Nuevamente, esa reacción posiblemente signifique una apelación indirecta a los criterios de verdad o de rectitud de lo dicho por el docente en el contexto escolar. Los intercambios entre las niñas y su maestro se dieron en el contexto de una clase sobre los cambios históricos y la modernidad. ${ }^{53}$

53 Los ejemplos se tomaron de un análisis más completo, en Rockwell (1995). 
1) M: Para tener contento a su dios... tenían que hacerle sacrificios humanos... y para tener contento a nuestro dios... en la actualidad... ¿hay que sacrificar personas? As: (seguras) No.

2) M: ... aquí (señalando en el libro) un médico naturista está curando con hierbas, ahora ya no se ha seguido el mismo sistema, no, ya cambió... ¿los médicos ahora curan todavía con hierbas?

As: (Silencio.) [Todavía curan con hierbas en la región.]

M: No.

3) M: ¿Era justo que unas cuantas gentes poseyeran muchas tierras y los demás fueran trabajadores?

As: (Silencio. Una alumna dice 'no', en voz baja.)

M: En cambio ahora... ¿Todos podemos ser dueños de tierras?

As: (Silencio. Unas afirman con la cabeza. Otra dice 'sî', en voz baja.)

M: Sí (voz firme), todos podemos... si algunos tienen más terrenitos es porque los han comprado, pero otros que no tienen la pueden comprar... en ese tiempo sólo los nobles podían comprar y tener tierras.

En los casos 2 y 3 debe ser comprensible para los alumnos el contenido proposicional, y es posible inferir la petición específica que les hace el maestro, ya que la estructura es idéntica a las preguntas que ha hecho numerosas veces en la misma clase (como en la secuencia 1). No obstante, en estos dos momentos ( 2 y 3 ) hay reticencia por parte de las alumnas. Sugiero interpretar esos momentos en que se detienen como una apelación al principio de verdad. Dudan de la afirmación que implícitamente solicita el maestro porque su experiencia no coincide con lo dicho. En ese caso no opera una transferencia automática de una forma de validación a otra (la norma de que lo que el maestro dice es verdad por ser maestro), por tratarse de un contenido que es accesible a la experiencia de las niñas. Esa interpretación se ve apoyada en el ejemplo 3 por la respuesta del maestro, quien agrega a lo que ha afirmado una autocorrección (“... la pueden comprar ...”), en aparente reconocimiento de la duda que expresan las alumnas, mediante su silencio, frente a su afirmación anterior de que "todos podemos ser dueños de tierras", realidad lejana para algunas de ellas.

Al plantear esa perspectiva basada en la teoría de Habermas, no niego la pertinencia del conocimiento cultural o de la dinámica conversacional. Propongo que puede ser importante usar la estructura común de la acción comunicativa (en lugar de un modelo de diferencias 
culturales y contextuales) para explicar la naturaleza de la resistencia. Es posible que ni las normas de rectitud ni los criterios empíricos de la verdad propios del maestro y de las alumnas sean idénticos. Lo que sí tienen en común es la posibilidad de invocar y de cuestionar tanto los criterios de verdad como las normas de rectitud en cualquier acción comunicativa. Así, pueden resistir la obligación, implícita en el acto de habla, de responder, de obedecer una orden o de aceptar como verdadero el contenido propuesto, si no consideran que se cumplieron las pretensiones de validez. Por supuesto, ello no responde a un razonamiento consciente, sino a los complejos procesos cognitivos y sociales involucrados en la competencia comunicativa de cualquier hablante.

Estoy convencida de que esa forma de resistencia se encuentra potencialmente presente en toda la comunicación escolar. Esta mirada torna visible la disyuntiva entre la "lógica de la interacción" y la "lógica del contenido" que los alumnos enfrentan continuamente en clase. Si ellos escogen seguir la "lógica de la interacción" deben aceptar como correcto y verdadero todo lo que pide y dice el maestro, por el hecho de ser maestro. Si intentan apropiarse de la "lógica del contenido", de encontrarle sentido al conocimiento escolar, tienden a relacionar y confrontar lo dicho por el maestro con su propia experiencia y pensamiento, lo cual los puede llevar a cuestionar lo que se les presenta en clase. No se trata de decidir si tienen o no la razón en esos momentos de desacuerdo, simplemente de reconocer que pueden dudar el carácter verdadero de lo que les dice el maestro.

La tensión entre esas dos lógicas se puede percibir en muchas ocasiones, aunque no siempre emerge al nivel discursivo por la naturaleza asimétrica y estratégica de gran parte de la interacción escolar. Los estudiantes, por el mero hecho de ser hablantes de una lengua, tienen la capacidad de interpretar, de inferir y de apelar a los criterios de verdad y rectitud. Esa fuerza explica una resistencia potencial que no es autocondenadora, una resistencia en que los alumnos no se "niegan a aprender" sino que invocan su derecho a cuestionar al maestro, e incluso a "indignarse" por lo que les dice o pide. ${ }^{54}$

Finalmente, es importante reconocer que el análisis del discurso es insuficiente para comprender las resistencias que se expresan en la

54 Desde luego, los maestros tienen la posibilidad de resistirse sobre esas bases frente a lo dicho por sus alumnos, o incluso por sus propias autoridades. 
interacción en el aula. Es necesario estudiar los contextos culturales, las relaciones sociales y las condiciones materiales que envuelven el discurso. En cualquier situación existen mecanismos efectivos de coerción que delimitan el campo de resistencias posibles. Las condiciones materiales de la escolarización (como el marco espacial y temporal del encuentro diario entre maestros y alumnos, o la exigencia de la certificación escolar para poder "vender" la fuerza de trabajo) también tienen efectos sobre la producción discursiva en el aula. Frente a esas condiciones, tanto maestros como estudiantes despliegan estrategias para asegurar su supervivencia dentro del contexto de trabajo y de estudio que es la escuela, o bien para resistir las exigencias escolares y construir alternativas.

\section{Reflexiones}

La discusión anterior apoyaría un replanteamiento de los procesos de resistencia en situaciones educativas. En la concepción más difundida, se considera que ciertas orientaciones comunicativas de grupos subordinados, que pueden ser fuente de resistencia frente a las pautas culturales o comunicativas impuestas por la escuela, contribuyen a la reproducción de las relaciones de dominación de una clase sobre otra. Una perspectiva distinta acerca de la comunicación, basada en la pragmática, apoyaría la búsqueda de criterios para distinguir actos de resistencia que no reproducen esas relaciones, sino que tienden a trascenderlas.

En la primera concepción, la reproducción desigual se atribuye a la socialización primaria y la consecuente interiorización de pautas culturales, comunicativas o cognitivas diferentes respecto a lo requerido por la escuela. Una variante la sitúa en la producción de una contracultura escolar que marca diferencias radicales frente a la cultura escolar. Se considera que la "no comunicación" resultante es suficiente garantía para perpetuar las distancias y relaciones entre categorías y clases sociales. En el fondo, la posición es similar al argumento de que la carencia de una "competencia comunicativa" específica (la formulación correcta del contenido) condena al alumno al fracaso escolar.

En la concepción alternativa, el peso de la reproducción diferencial se localiza en los mecanismos escolares, así como en la estructura social en su conjunto. Sin duda, la posición social de las clases dominadas, si bien puede implicar tradiciones culturales y comunicativas diferentes, genera distintas oportunidades materiales de apropiación del conocimiento 
y de su uso legítimo, lo cual desde luego se refleja en contextos escolares y afecta el destino escolar de los alumnos. No obstante, esa posición no necesariamente implica una competencia comunicativa deficiente que lleve a una resistencia autocondenadora.

Las reglas específicas de diferentes situaciones escolares no siempre corresponden de manera homogénea a "un código elaborado". Si bien puede haber múltiples tradiciones comunicativas entre los estudiantes, las reglas escolares también generan diferencias y son constitutivas de las relaciones de poder; no son neutrales. El ejercicio del poder, que tantas veces excluye de la cultura escrita a los niños de las clases llamadas trabajadoras o populares, supone procesos más activos generados desde la propia escuela y la sociedad. Esos procesos contrarrestan una resistencia potencial en la cual los alumnos ponen en juego no sólo diferencias culturales sino también estructuras lingüísticas y cognitivas comunes a todo ser humano. Con esas capacidades culturales y comunicativas, los estudiantes participan en la interacción escolar de manera más dinámica de lo que suponen las nociones de una competencia comunicativa deficiente o un código restringido. Siempre se entretejen en la trama de la reproducción procesos de apropiación real del conocimiento y de resistencia frente a la discriminación social. Siempre se abre la posibilidad de una verdadera indignación (y no sólo una oposición) frente a determinados actos — incluyendo actos de habla — que ocurren en espacios escolares.

\section{Referenclas}

ANYON, Jean. Social class and school knowledge. Curriculum Inquiry, Ontario, ciudad, v. 11, n. 1, p. 3-42, 1981.

APPLE, Michael. Reproduction, contestation and curriculum: an essay in selfcriticism. Interchange, Ontario, v. 12, n. 2-3, p. 27-47, June 1981.

ATKINSON, Paul. Inspecting classroom talk. In: ADELMAN, C. (Ed.). Uttering, muttering: collecting, using and reporting talk for social and educational research. London: Grant McIntyre, 1981. p. 98-113.

AUSTIN, John L. How to do things with words. Oxford: Oxford University Press, 1962.

BERNSTEIN, Basil. Codes, modalities, and the process of cultural reproduction: a model. Language in Society, Cambridge, v. 10, n. 3, p. 327-363, July 1981.

v. 1 y 3 .

Class, codes and control. London: Routledge y Kegan Paul, 1971 y 1975. 
BOURDIEU, Pierre. Cosas dichas. Traducción de Margarita Mizraji. Barcelona: Gedisa, 1988. (Título original: Choses dites).

BOURDIEU, Pierre; PASSERON, Jean Claude. La reproducción. Elementos para una teoría del sistema de enseñanza. Barcelona: Laia, 1981.

CANDELA, María Antonia. Poder en el aula: una construcción situacional. Discurso, Teoria y Análisis, México, n. 23-24, p. 23-24, 139-157, 2001.

Ciencia en el aula: los alumnos entre la argumentación y el consenso. México: Paidós, 1999.

CAZDEN, Courtney. Classroom discourse: the language of teaching and learning. Portsmouth, New Hampshire: Heinemann, 1988.

CAZDEN, Courtney; JOHN, Vera P.; HYMES, Dell (Ed.). Functions of language in the classroom. New York: Teachers' College Press, 1972.

CICOUREL, Arnold et al. Language use and school performance. Chicago: Academic Press, 1974.

CONNELL, Robert W. et al. Making the difference: school, families and social division. London: George Allen and Unwin, 1982.

DURANTI, Alessandro. Antropología lingüistica. Barcelona: Cambridge University Press, 2000.

EDWARDS, Derek; MERCER, Neil. Common knowledge: the development of understanding in the classroom. London: Routledge and Kegan Paul, 1987.

ERICKSON, Frederick. Qualitative methods of research in teaching. In: WITTROCK, M. C. (Ed.). Handbook of research on teaching. $3^{\text {rd }}$ ed. New York: McMillan, 1986. p. 119-161.

Classroom discourse as improvisation: the relationship between academic task structure and social participation structure in lessons. In: WILKENSON, L. Ch. (Ed.). Communicating the classroom. New York: Academic Press, 1982. p. 153-181.

Going for the zone: the social and cognitive ecology of teacher-student interaction in classroom conversations. In: HICKS, D. (Ed.). Discourse, learning and schooling. New York: Cambridge University Press, 1996. p. 29-62.

EVERHART, Robert B. Reading, writing and resistance: adolescence and labor in a junior high school. Boston: Routledge and Kegan Paul, 1983.

FOLEY, Douglas. Learning capitalist culture: deep in the heart of Tejas. Philadelphia: University of Pennsylvania Press, 1990.

GIROUX, Henry. Teorías de la reproducción y la resistencia en la nueva sociología de la educación: un análisis crítico. Cuadernos Políticos, México, n. 44, p. 33-65, jul./ dic. 1985. 
GUMPERZ, John J. Discourse strategies. New York: Cambridge University Press, 1982.

GUTIERREZ, Kris; RYMES, B.; LARSON, J. Scripts, counterscripts, and underlife in the classroom: James Brown versus "Brown v. Board of Education". Harvard Educational Review, Cambridge, M. A., v. 65, n. 3, p. 445-471, 1995.

HABERMAS, Jürgen. Teoría de la acción comunicativa. Racionalidad de la acción y racionalización social. Buenos Aires: Taurus, 2000. v. 1.

HAMMERSLEY, Martyn. School learning: the cultural resources required by pupils to answer a teacher's question. In: WOODS, P.; HAMMERSLEY, M. (Ed.). School experience: explorations in the sociology of education. New York: St Martin's Press, 1977. p. 57-86.

HARGREAVES, Andy. The significance of classroom coping strategies. In: BARTON, L.; MEIGHAN, R. (Ed.). Sociological interpretations of schooling and the classroom. Nafferton, Driffield, England: Nafferton Books, 1978. p. 73-100.

HEATH, Shirley Brice. Ways with words: language, life and work in communities and classrooms. New York: Cambridge University Press, 1983.

HICKS, Deborah (Ed.). Discourse, learning and schooling. New York: Cambridge University Press, 1996.

HYMES, Dell. Language and education: ethnolinguistic essays. Washington, D. C.: Center for Applied Linguistics, 1980.

Introduction. In: CAZDEN, C.; JOHN, V.; HYMES, D. (Ed.). Functions of language in the classroom. New York: Teacher's College Press, 1972.

JACKSON, Philip W. Life in classrooms. New York: Holt, Rinehart and Winston, 1968.

LEVINSON, Bradley; HOLLAND, Dorothy. The cultural production of the educated person: an introduction. In: LEVINSON, B.; FOLEY, D.; HOLLAND, D. (Ed.). The cultural production of the educated person. Critical ethnographies of schooling and local practice. Albany: SUNY Press, 1996. p. 1-54.

LEVINSON, Stephen C. Pragmatics. New York: Cambridge University Press, 1983.

MARKOVÁ, Ivana; FOPPA, Klaus. Asymmetries in dialogue. Harvester, Wheatsheaf: Barnes and Noble Books, 1991.

McCARTY, Cameron; APPLE, Michael W. Race, class and gender in American educational research: toward a nonsynchronous parallelist position. In: WEIS, Lois (Ed.). Class, race, and gender in American education. Albany: State University of New York Press, 1988. p. 9-39. 
McDEMOTT, Ray P. Social relations as contexts for learning in school, Harvard Educational Review, Cambridge, M. A., v. 47, n. 2, p. 198-213, 1977.

MEHAN, Hugh. Learning lessons: social organization in the classroom. Cambridge: Harvard University Press, 1979.

PHILIPS, Susan U. Participant structures and communicative competence: warm springs children in community and classroom. In: CAZDEN, C.; JOHN, V. P.; HYMES, D. (Ed.). Functions of language in the classroom. New York: Teachers' College Press, 1972. p. 370-394.

RECANATI, François. La transparencia y la enunciación. Buenos Aires: Hachette, 1979.

ROCKWELL, Elsie. En torno al texto. In: ROCKWELL, E. (Coord.). La escuela cotidiana. México: Fondo de Cultura Económica, 1995. p. 198-222.

Palabra escrita, interpretación oral: los libros de texto en la clase. Infancia y Aprendizaje, Madrid, v. 14, n. 3, p. 29-43, sept. 1991.

Los usos escolares de la lengua escrita. In: FERREIRO, E.; GÓMEZ

PALACIO, M. (Comp.). Nuevas perspectivas sobre los procesos de lectura y escritura. México: Siglo Veintiuno, 1982. p. 296-320.

ROCKWELL, E.; GALVEZ, García. Formas de transmisión del conocimiento científico. Educación, México, v. 8, n. 42, p. 97-140, oct./dic. 1982.

SACKS, Harvey; SCHEGLOFF, Emmanuel A.; JEFFERSON, Gail. A simplest systematics for the organization of turn-taking in conversation. Language, Washington, D. C., v. 50, n. 4, part 1, p. 696-735, dec. 1974.

SEARLE, John R. Speech acts. London: Cambridge University Press, 1969.

SHARP, Rachel; GREEN, Anthony. Education and social control. London: Routledge and Kegan Paul, 1975.

SHARROCK, Wes; ANDERSON, Bob. The ethnomethodologists. London: Tavistock, 1986.

STUBBS, Michael. Discourse analysis: the sociolinguistic analysis of natural language. Chicago: The University of Chicago Press, 1983.

Language, schools and classrooms. London: Methuen, 1976.

TADEU DA SILVA, Tomas. Distribution of school knowledge and social reproduction in a Brazilian urban setting. British Journal of Sociology of Education, London, v. 9, n. 1, p. 55-79, Feb. 1988.

VALENTINEZ, María de la Luz. La persistencia de la lengua y la cultura purépecha frente a la educación escolar. México: Dirección General de Educación Indígena, Secretaría de Educación Pública, 1982. 
VARENNE, Hervé; McDERMOTT, Ray. Successful failure: the school America builds. Boulder: Westview Press, 1999.

WEIS, Lois. Class, race and gender in American education. Albany: State University of New York, 1988.

WILLIS, Paul. Cultural production is different from cultural reproduction is different from social reproduction is different from reproduction. Interchange, Ontario, v. 12, n. 2-3, p. 48-67, June 1981.

Learning to labour: how working class kids get working jobs. London: Saxon, 1977.

WOODS, Peter (Ed.). Teacher strategies: explorations in the sociology of the school. London: Croom Helm, 1980.

Recebido em: 22/09/2006

Aprovado em: 28/10/2006 